\title{
Care and feeding of frogs
}

\author{
Margaret $\operatorname{Pan}^{1}$, Eugene Chiang ${ }^{1,2}$ \\ mpan@astro.berkeley.edu
}

\begin{abstract}
"Propellers" are features in Saturn's A ring associated with moonlets that open partial gaps. They exhibit non-Keplerian motion (Tiscareno et al. 2010); the longitude residuals of the best-observed propeller, "Blériot," appear consistent with a sinusoid of period $\sim 4$ years. Pan \& Chiang (2010) proposed that propeller moonlets librate in "frog resonances" with co-orbiting ring material. By analogy with the restricted three-body problem, they treated the co-orbital material as stationary in the rotating frame and neglected non-co-orbital material. Here we use simple numerical experiments to extend the frog model, including feedback due to the gap's motion, and drag associated with the Lindblad disk torques that cause Type I migration. Because the moonlet creates the gap, we expect the gap centroid to track the moonlet, but only after a time delay $t_{\text {delay }}$, the time for a ring particle to travel from conjunction with the moonlet to the end of the gap. We find that frog librations can persist only if $t_{\text {delay }}$ exceeds the frog libration period $P_{\text {lib }}$, and if damping from Lindblad torques balances driving from co-orbital torques. If $t_{\text {delay }} \ll P_{\text {lib }}$, then the libration amplitude damps to zero. In the case of Blériot, the frog resonance model can reproduce the observed libration period $P_{\text {lib }} \simeq 4 \mathrm{yr}$. However, our simple feedback prescription suggests that Blériot's $t_{\text {delay }} \sim 0.01 P_{\text {lib }}$, which is inconsistent with the observed libration amplitude of $260 \mathrm{~km}$. We urge more accurate treatments of feedback to test the assumptions of our toy models.
\end{abstract}

\section{Introduction}

"Propellers" observed by the Cassini spacecraft in Saturn's A ring appear as S-like features superimposed on azimuthally long and radially narrow gaps (Tiscareno et al. 2006;

\footnotetext{
${ }^{1}$ Department of Astronomy, University of California, Berkeley, CA 94720

${ }^{2}$ Department of Earth and Planetary Science, University of California, Berkeley, CA 94720
} 
Sremčević et al. 2007; Tiscareno et al. 2008, 2010). Each propeller is believed to trace a moonlet several hundred meters in size which gravitationally repels nearby ring particles, creating an underdense gap in the moonlet's immediate vicinity, as well as overdensities at radii just outside the gap. Because of Keplerian shear, these density perturbations propagate toward greater longitudes inside the moonlet's orbit and smaller longitudes outside, producing two lobes separated by a few Hill spheres of the moonlet (Seiß et al. 2005; Lewis \& Stewart 2009). The gap's azimuthal length is set by the moonlet mass and the time for ring particles to diffuse back into the gap via particle-particle interactions (Spahn \& Sremčević 2000; Sremčević et al. 2002; Seiß et al. 2005; Lewis \& Stewart 2009).

Propellers in the outer A ring exhibit non-Keplerian motion (Tiscareno et al. 2010). In particular, the longitude residuals of the propeller "Blériot," observed 89 times over 4.2 years, show variations consistent with a sine wave of half-amplitude $260 \mathrm{~km}$ and period 3.68 years. The longitude residuals imply semimajor axis variations of order $100 \mathrm{~m}$. These data constrain the underlying mechanism. The variations' smooth sinusoidal character suggests that this mechanism is not stochastic on timescales shorter than a few years. Secular interactions with the Saturnian moons, rings, or equatorial bulge have timescales typically much longer than a few years; in any case, secular interactions cannot induce semimajor axis changes. Finally, no other Saturnian moon appears to occupy a mean motion resonance with Blériot (Tiscareno et al. 2010).

Pan \& Chiang (2010, hereafter PC) proposed that Blériot's non-Keplerian motion is caused by gravitational interactions between Blériot's moonlet and co-orbital ring material outside the moonlet's gap. The interaction is long-range: the co-orbital mass is located thousands of Hill sphere radii away from the moonlet. The propeller moonlet and the much more massive co-orbital material participate in a 1:1 resonance reminiscent of tadpole and horseshoe orbits in the conventional restricted three-body problem. The moonlet performs what PC called "frog" librations within the gap.

To re-cap the main ideas behind PC's analysis we give the following order-of-magnitude description of frog librations. Because our goal here is to describe the physical processes clearly, we neglect factors of order unity; we refer readers to $\S 2$ of PC for an exact treatment. We use units where Saturn has mass $M_{\text {Saturn }}=1$, the co-orbital mass's distance from Saturn is $r_{\text {coorb }}=1$, and Newton's constant $G$ is 1 . We work in the frame co-rotating with a test particle on a circular orbit of radius 1 and (as a consequence of our unit system) angular velocity 1 . The moonlet of mass $\mu_{\text {moon }}$ opens a gap of angular size $2 \phi \ll \pi$ in the co-orbital material (Figure 1). The co-orbital material outside the gap has mass $\mu_{\text {ring. Since the co- }}$ orbital material closest to the ends of the gap interacts most strongly with the moonlet, we model the co-orbital material as two point masses, each of mass $\mu_{\text {end }}=\mu_{\text {ring }} \phi / 2$, fixed at 
longitudes $\pm \phi$. We label the moonlet's polar coordinates $(r=1+\Delta, \theta)$ where $\Delta \ll 1$ and $|\theta| \ll \phi$. The moonlet librates about the equilibrium point 11 between the co-orbital masses, as shown in the right-hand side of Figure 1,

The moonlet's motion is governed by Keplerian shear and gravitational interactions with the co-orbital masses. We derive scaling relations for the frog libration's period and radial/azimuthal aspect ratio as follows. The azimuthal length $\theta_{\max }$ of one libration is of order the moonlet's azimuthal Keplerian drift in one libration period $P_{\text {lib. }}$ If the libration radial width is $\Delta_{\max }$, then the moonlet's drift speed is of the same order, and

$$
\theta_{\max } \sim P_{\text {lib }} \cdot \Delta_{\max }
$$

Over the libration cycle, gravitational interactions with the co-orbital masses change the moonlet's semimajor axis from $1+\Delta_{\max }$ to $1-\Delta_{\max }$. The corresponding fractional change in the moonlet's specific angular momentum is $\sim \Delta_{\max }$. The angular momentum changes because the (azimuthal) gravitational forces $\sim \pm \mu_{\text {end }} / \phi^{2}$ exerted by the co-orbital masses do not cancel exactly; the residual is of order $\theta / \phi$ :

$$
\Delta_{\max } \sim \frac{\mu_{\mathrm{end}}}{\phi^{2}} \frac{\theta_{\max }}{\phi} \cdot P_{\mathrm{lib}}
$$

Together, Eqs. 1 and 2 imply

$$
P_{\text {lib }} \sim \frac{\phi^{3 / 2}}{\mu_{\text {end }}^{1 / 2}} \quad, \quad \frac{\Delta_{\max }}{\theta_{\max }} \sim \frac{\mu_{\text {end }}^{1 / 2}}{\phi^{3 / 2}} .
$$

These scalings match those of the exact relations in Eqs. 10 and 11 of PC, which we repeat here for reference:

$$
P_{\text {lib }}=\frac{\pi}{\sqrt{3}} \frac{\phi^{3 / 2}}{\mu_{\text {end }}^{1 / 2}} \quad, \quad \frac{\Delta_{\max }}{\theta_{\max }}=\frac{4}{\sqrt{3}} \frac{\mu_{\mathrm{end}}^{1 / 2}}{\phi^{3 / 2}} .
$$

Note that the coorbital mass $\mu$ used by PC equals $2 \mu_{\text {end }}$ as we define it here.

In a more detailed calculation with the co-orbital mass spread uniformly over the entire co-orbital region outside the gap, PC confirmed the scalings of Eq. 3 and found that for parameters characteristic of Blériot's environment, the frog librations should indeed have a period of $\sim 4$ years.

While the agreement between the period of the frog resonance as estimated by PC, and the period of Blériot's longitude variations as observed by Tiscareno et al. (2010) is

\footnotetext{
${ }^{1}$ This equilibrium point is a local maximum of the gravitational potential. The moonlet can librate about this potential maximum because the Coriolis force stabilizes its motion.
} 


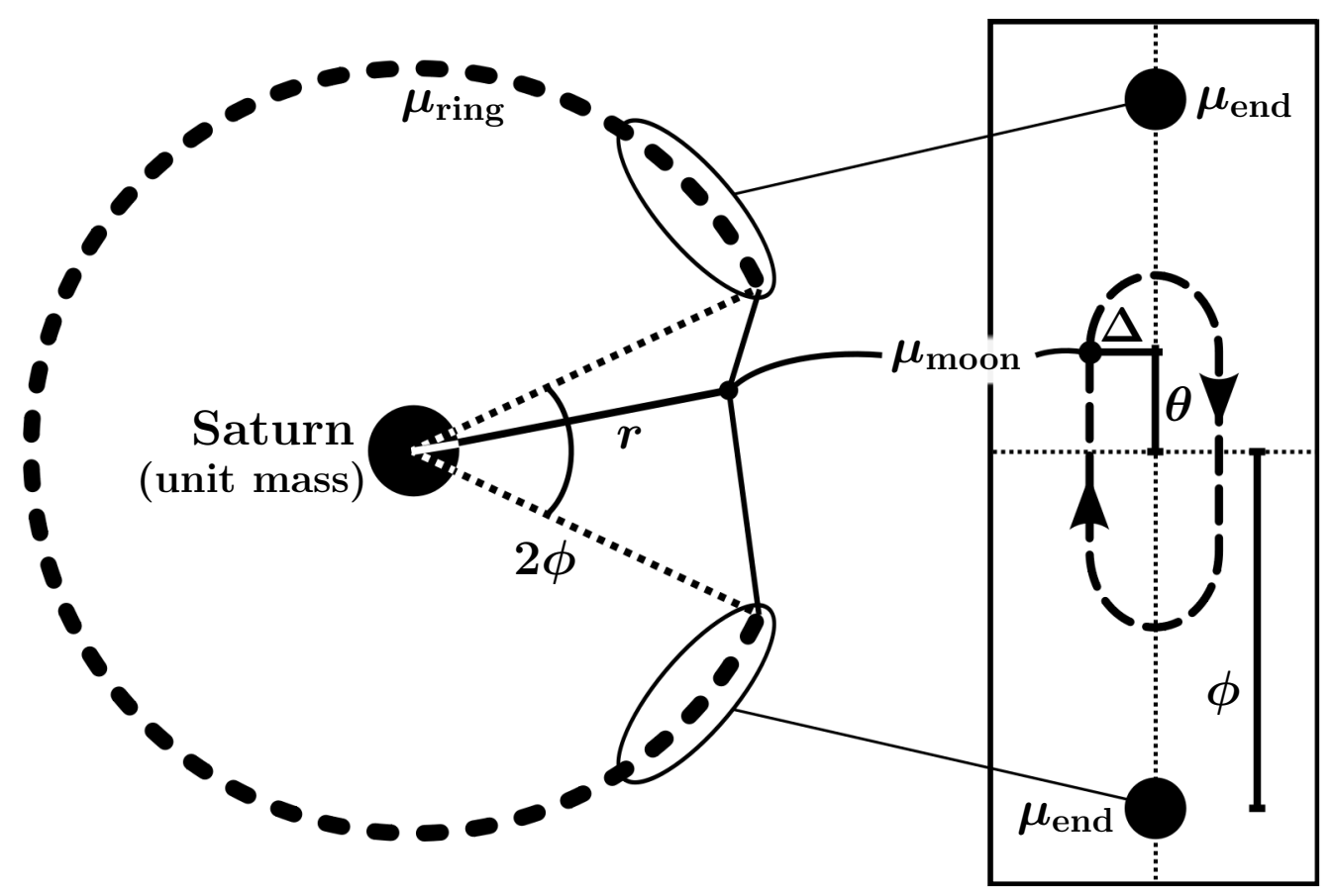

Fig. 1. - Schematic of frog librations. The left side shows the geometric configuration of the moonlet and its co-orbital material. We use units where Saturn's mass is unity and where the orbit radius of the co-orbital ring material (dashed circle arc) is unity. The moonlet of mass $\mu_{\text {moon }}$ located at polar coordinates $(r=1+\Delta, \theta)$ moves in its gap of angular size $2 \phi$ in the co-orbital material. The portions of the co-orbital mass $\mu_{\text {ring }}$ that interact most strongly with the moonlet are those within azimuth $\sim \phi$ of the ends of the moonlet's gap. For simplicity, we model the entire co-orbital mass as two identical point masses of mass $\mu_{\text {end }}=\mu_{\text {ring }} \phi / 2$ located at the gap ends. The dashed oval curve on the right represents a frog libration trajectory. 
promising, PC's treatment is incomplete. In particular, PC treated the co-orbital masses as stationary (in the co-rotating frame). This is a severe simplification. Because the moonlet creates and maintains its gap, the co-orbital material must, to some degree, follow the moonlet's position. If it follows too closely, the frog libration amplitude $(\theta)$ may be unobservably small. On the other hand, if the gap ends are far enough away from the moonlet, the mass there will respond sluggishly to the moonlet's motion because of the finite time needed for particles to drift from conjunction with the moonlet to the gap ends. In this case, large-amplitude frog librations should be permitted.

A self-consistent understanding of the moonlet's motion must account for how the nonKeplerian motions of the moonlet feed back into the non-Keplerian motions of the co-orbital masses. Here we investigate the behavior of frog orbits when the co-orbital masses move in response to the moonlet, and when Lindblad torques due to close encounters between the moonlet and ring particles are present. Our focus here is on conceptual clarity; from a technical standpoint, our models are, by design, crude. Many of our calculations are accurate to order-of-magnitude at best. In our equations we use " " to denote a relation in which order-unity factors are ignored; " $\simeq$ " to denote a relation in which order-unity factors are retained but some quantities remain approximate or not precisely defined; and "=" to denote an exact relation. In Section 2.1 we present a simple toy model for feedback and examine its implications for frog librations. In Section 2.2 we add Lindblad torques. In Section 3 we summarize and comment on the results of our experiments.

\section{Modeling the gap motion and Lindblad torques}

Figure 2 gives an overview of the formation and maintenance of the moonlet gap. The moonlet sits in a sea of much smaller ring particles with mass surface density $\Sigma$. In close encounters with ring particles, the moonlet gravitationally repels the particles, clearing a

region whose initial radial size is of order the moonlet's Hill sphere $\mu_{\text {moon }}^{1 / 3}$. As repelled particles drift away in longitude from the moonlet because of Keplerian shear, they collide with other particles; over a time $t_{\text {delay, }}$ they diffuse back into the gap, refilling it. Over this diffusion time, the perturbed ring particles drift an angular distance $\phi$ from their close encounter with the moonlet to the end of the gap. That is,

$$
t_{\text {delay }} \simeq \frac{2}{3} \frac{\phi}{x_{\text {gap }}}
$$

where $x_{\text {gap }}<\mu_{\text {moon }}^{1 / 3}$ is the gap's typical downstream radial half-width (radial distance between the moonlet's orbit and one edge of the gap). In this definition $x_{\text {gap }}$ is the gap width along most of the gap's (azimuthal) length, far downstream of the moonlet's location; its value 
depends on the way ring particles diffuse back into the gap and, therefore, on the local ring viscosity. The downstream gap width $x_{\text {gap }}$ may differ from the gap width $x \sim \mu_{\text {moon }}^{1 / 3}$ at the moonlet's longitude.

We can think of $t_{\text {delay }}$ as the delay between any movement of the moonlet and the response of the gap ends to that movement. If the moonlet were to suddenly shift its position in the rotating frame, the gap ends would re-position themselves so as to be centered on the moonlet's new position - but only after a time $t_{\text {delay }}$. This simple picture suggests that we modify the model of PC so that at any given instant, the co-orbital masses are located $\pm \phi$ in longitude away from the moonlet's location a time $t_{\text {delay }}$ ago.

\subsection{Experiments including gap motion only}

To explore the effects of this delayed gap motion on the moonlet, we construct a numerical toy model. As in PC, we treat the co-orbital material as two point masses each of mass $\mu_{\text {end }}$ located at the ends of the moonlet gap, and we integrate the trajectory of the moonlet (test particle) as it interacts with the point masses and the central unit mass. To model the feedback, we fix the point masses' angular separation to be $2 \phi$ and their radial coordinate to be unity, and force them to move so that the longitude of their midpoint at time $t$ equals the moonlet's longitude at time $t-t_{\text {delay }}$. To begin the calculation, we integrate from $t=0$

to $t=t_{\text {delay }}$ with stationary point masses, and then use this history when we turn on the feedback at time $t=t_{\text {delay }}$. We treat $\mu_{\text {end }}, \phi$, and $t_{\text {delay }}$ as free parameters.

The top panel of Figure 3 shows representative results from this toy model. When $t_{\text {delay }}$ is short compared to the nominal frog libration time (Eq. 4), the librations damp quickly. When $t_{\text {delay }}$ is comparable to $P_{\text {lib }}$ or longer, the libration amplitude grows and the moonlet eventually exits the resonance. The transition between amplitude growth and amplitude decay occurs when $P_{\text {lib }}$ is a few times $t_{\text {delay }}$; in the representative example shown in Figure 3 , this transition occurs when $t_{\text {delay }} \sim P_{\text {lib }} / 3$.

The shape of the potential the moonlet experiences also changes because of the co-orbital masses' motion. The change in the potential alters $P_{\text {lib }}$ in Figure 3, $P_{\text {lib }} \simeq 60$ rather than $P_{\text {lib }} \simeq 81.6$ as predicted by Eq. 4. In our numerical trials using parameter values spanning the ranges $10^{-7}<\mu_{\text {end }}<10^{-4}, 10^{-3}<\phi<0.5$, and $10<t_{\text {delay }}<7000$, we found that the ratio between $t_{\text {delay }}$ and $P_{\text {lib }}$ is the principal deciding factor for the moonlet's qualitative behavior.

We interpret the growing librations when $t_{\text {delay }} \gtrsim P_{\text {lib }}$ as resonant forcing. In this case, the gap position lags the moonlet position by a phase of order unity or, equivalently, a 


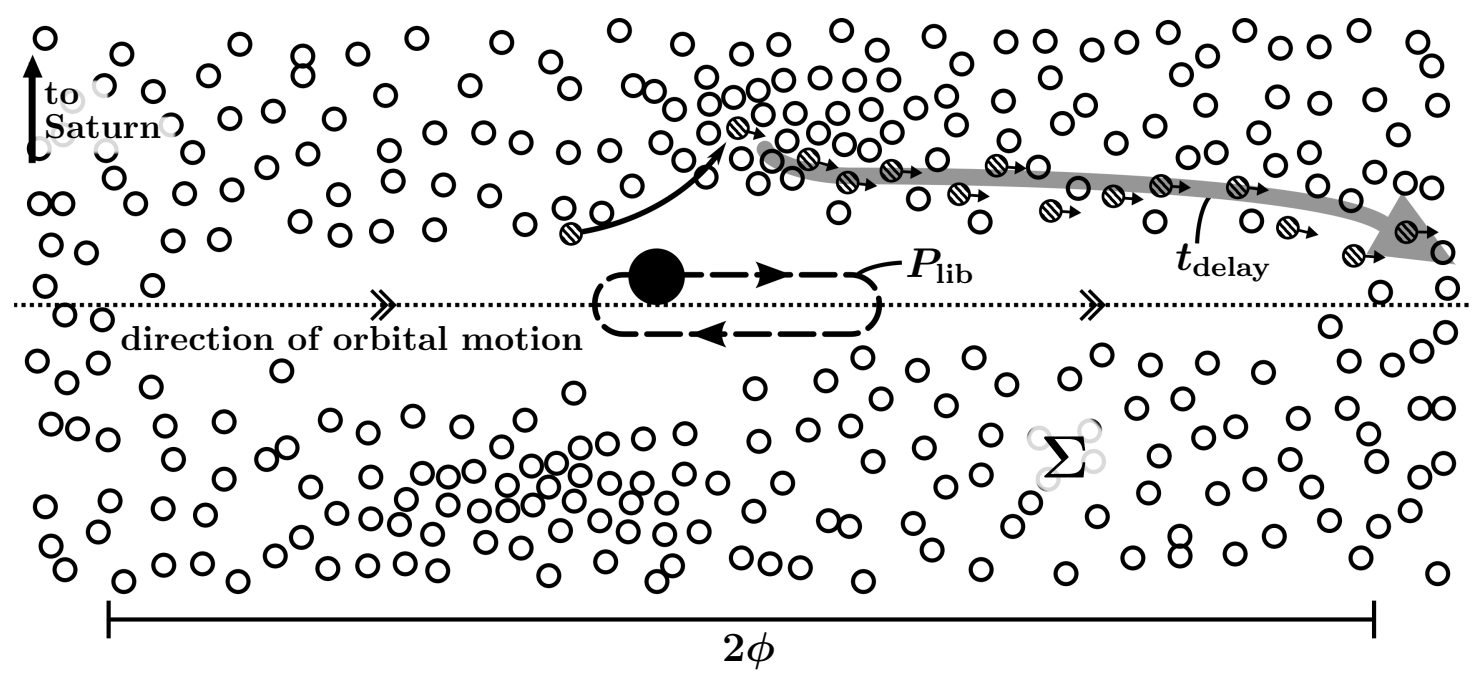

Fig. 2. - Schematic showing the trajectory of a ring particle (shaded circle with arrow) participating in gap formation. A moonlet of mass $\mu_{\text {moon }}$ is embedded in a particle disk of surface density $\Sigma$. For a particle with initial radius inside the moonlet's orbit (toward the top of the figure), a gravitational interaction with the moonlet decreases the particle's semimajor axis. Diffusion via collisions with nearby ring particles moves the particle back to its original semimajor axis over a time $t_{\text {delay }}$. During this time the particle drifts an angle $\phi$ relative to the moonlet because of Keplerian shear. The dashed oval represents frog librations of the moonlet with period $P_{\text {lib }}$. 
longitude of order the libration amplitude. Because the co-orbital masses are slaved to the moonlet's actual past motion, they automatically drive the moonlet at resonance.2

In the limit $t_{\text {delay }} \ll P_{\text {lib }}$, however, the moonlet's displacement from the gap center is always small compared to the libration amplitude that a moonlet with the same location and velocity would have if the co-orbital masses were stationary. The moonlet's speed relative to the gap is also much less than the speed it would have if the co-orbital masses were stationary. Continuously shrinking the moonlet's displacement from the equilibrium point between the co-orbital masses, and its speed relative to the equilibrium point, damps the libration amplitude until the moonlet lands at the fixed point of the potential.

We find that the transition between the regimes of growing and decaying libration amplitude occurs over a narrow range in $t_{\text {delay }}$. For example, the values for $t_{\text {delay }}$ corresponding to the two different outcomes in the top panel of Figure 3 differ by less than $10 \%$ of $P_{\text {lib. }}$.

We performed some supplementary experiments. First we forced the co-orbital masses to move sinusoidally in longitude with prescribed amplitude and frequency. Setting the co-orbital masses' oscillation period equal to $P_{\text {lib }}$ as given by Eq. 4 produces beats in the moonlet's motion: the motion of the co-orbital masses changes the shape of the potential the moonlet sees, shifting the libration period enough for the driving to be just off resonance (Figure 4). If we decrease the co-orbital masses' oscillation amplitude while keeping their oscillation period fixed, the potential experienced by the moonlet changes less. Then the driving is closer to resonance and the beat frequency decreases. Compare these results with those of our original experiments, where the co-orbital masses were slaved to the moonlet's actual past motion and the driving was therefore exactly on resonance.

In experiments aimed at softening the transition between the regimes of growing and decaying amplitude, we performed simulations where we artificially weakened the feedback strength. To do this we forced the co-orbital masses to follow the moonlet's past motion, as before, but scaled the amplitude of the co-orbital masses' motions relative to the moonlet's by a constant factor. We observed no qualitative change in the moonlet's behavior. The value of $t_{\text {delay }}$ at the transition changed by a factor of order unity.

To summarize our findings so far: the libration amplitude damps to zero if $t_{\text {delay }}$ is too short and grows without bound if $t_{\text {delay }}$ is comparable to or longer than $P_{\text {lib }}$, with an abrupt transition between the two regimes. However, longitude residuals like those observed might

\footnotetext{
${ }^{2}$ Since the co-orbital masses follow the moonlet's past rather than its current motion, the driving frequency may, in principle, differ slightly from resonance, particularly for $t_{\text {delay }} \gg P_{\text {lib }}$. However, the driving frequency always moves toward resonance with time.
} 
be produced in the latter regime if some other mechanism damps the motion enough to limit the libration amplitude's growth. We explore one possible damping mechanism in $₫ 2.2$.

The requirement that $t_{\text {delay }} \gtrsim P_{\text {lib }} / 3$ that we find in our simulations (the factor of 3 is empirical) has a simple consequence for the gap's radial width: using Eq. 4 and $\mu_{\text {end }} \simeq$ $\Sigma \cdot 2 x_{\text {gap }} \cdot \phi$, we find that

$$
t_{\text {delay }} \gtrsim \frac{\pi}{3 \sqrt{3}} \frac{\phi^{3 / 2}}{\mu_{\text {end }}^{1 / 2}} \simeq \frac{\pi}{3 \sqrt{6}} \frac{\phi}{\sqrt{\sum x_{\text {gap }}}}
$$

which together with Eq. 5 implies that

$$
\frac{x_{\text {gap }}}{r_{\text {coorb }}} \lesssim \frac{24}{\pi^{2}} \frac{\Sigma r_{\text {coorb }}^{2}}{M_{\text {Saturn }}}
$$

or

$$
x_{\text {gap }} \lesssim 4 \mathrm{~m}\left(\frac{\Sigma}{40 \mathrm{~g} / \mathrm{cm}^{2}}\right)\left(\frac{r_{\text {coorb }}}{1.34 \times 10^{10} \mathrm{~cm}}\right)^{3}
$$

where for the last line we have restored the units and used numbers inspired by Blériot and the A ring (Tiscareno et al. 2010; Colwell et al. 2009).

At face value, Eq. 8 suggests that for Blériot's moonlet to avoid libration damping, its gap should be radially narrow, of order several meters across, over a significant portion of its azimuthal extent. The required narrowness seems implausible, as it is comparable in size to the meter-sized particles dominating the mass in the A ring (see Table 15.1 of Cuzzi et al. 2009). Thus, Eq. 8 argues against frog librations as being the correct explanation for the observed non-Keplerian motions of propellers. We stress, however, that our equations are only as accurate as the toy model for $t_{\text {delay }}$ from which they derive. A more careful analysis of the gap's reactions to the moonlet (e.g., using numerical simulations of the entire gap) would provide an important test of our estimates here.

\subsection{Experiments including gap motion and Lindblad torques}

The results of \$2.1 indicate that stable frog librations can persist only if $t_{\text {delay }} \gtrsim P_{\text {lib }}$ and if some other mechanism damps the librations enough to stop their runaway growth. One damping mechanism is disk torques due to Lindblad resonances - the same torques that open the gap and are responsible for Type I migration. Because Lindblad torques repel the moonlet away from the gap edges, forcing the moonlet's semimajor axis back toward the gap center, we expect such torques to decrease the frog libration amplitude.

To provide a conceptual basis for the behavior we expect in our numerical experiments, we give the following order-of-magnitude estimates of the Lindblad torques and associated 

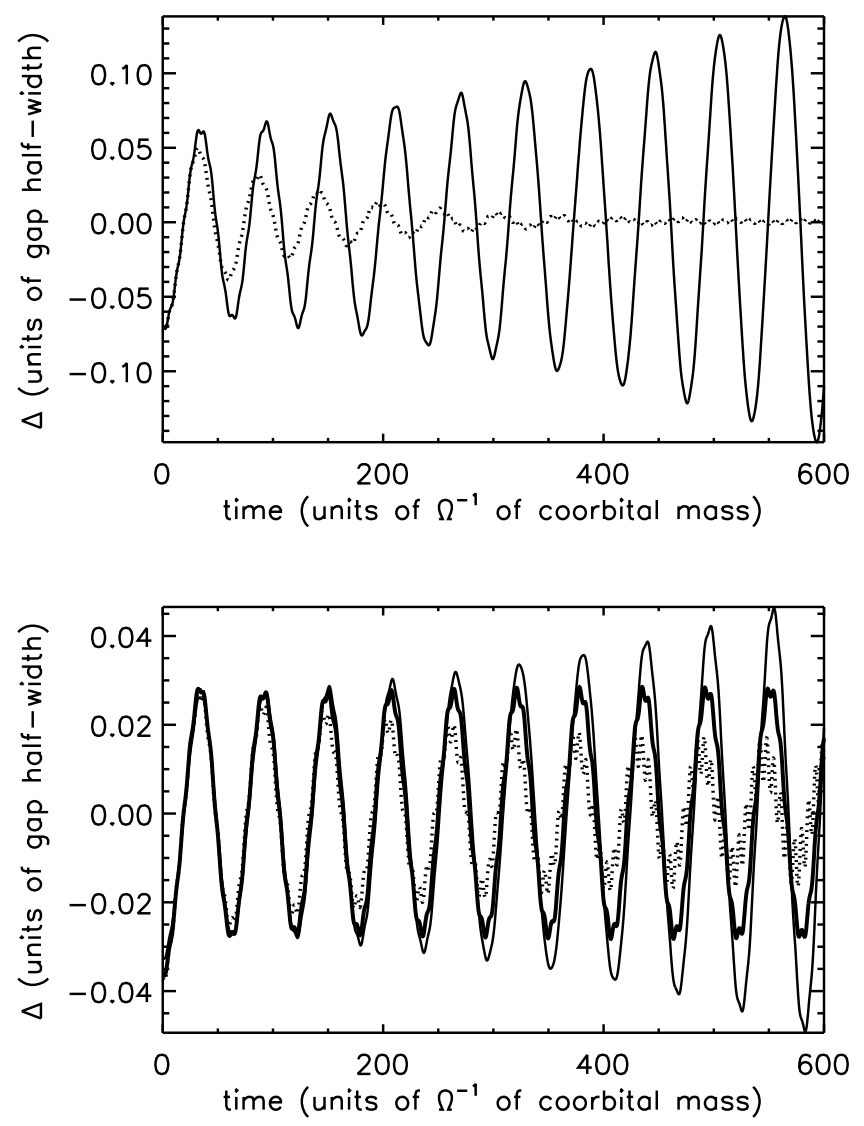

Fig. 3.- Sample moonlet trajectories showing the effects of feedback from co-orbital mass motion and damping from Lindblad torques. The parameter values $\mu_{\text {end }}=10^{-4.5}$ and $\phi=0.4$ are used throughout, so that the nominal libration period according to Eq. 团 is $P_{\mathrm{lib}}=81.6$. Including co-orbital mass motion changes the potential the moonlet sees; in all the sample trajectories shown here, the libration period in the altered potential is actually $P_{\text {lib }} \simeq 60$. The top panel shows the effects of co-orbital mass motion alone with different values of $t_{\text {delay }}$, the time by which the co-orbital mass motion is delayed relative to the moonlet's motion (i.e., the gap re-adjustment time). Values of $t_{\text {delay }}$ greater than some threshold lead to increasing libration amplitudes (solid curve, $t_{\text {delay }}=30$ ). Values of $t_{\text {delay }}$ even slightly smaller than the threshold produce decreasing amplitudes (dotted curve, $t_{\text {delay }}=25$ ). The bottom panel includes the effects of both gap movement and Lindblad disk torques. All three trajectories have $t_{\text {delay }}=30$ and $\Sigma=0.01$, but the moonlet masses are $\mu_{\text {moon }}=\{1.48,7.41,37.1\} \times 10^{-7}$, respectively, for the light solid, heavy solid, and dotted curves, and the gap widths are $x=6$ moonlet Hill radii. The coefficient of 6 is given approximately by the edges of the propeller gaps computed by Spahn \& Sremčević (2000). The trajectories shown here are representative of our numerical experiments and are meant to illustrate our findings; the parameter values used do not correspond to the propeller Blériot. 


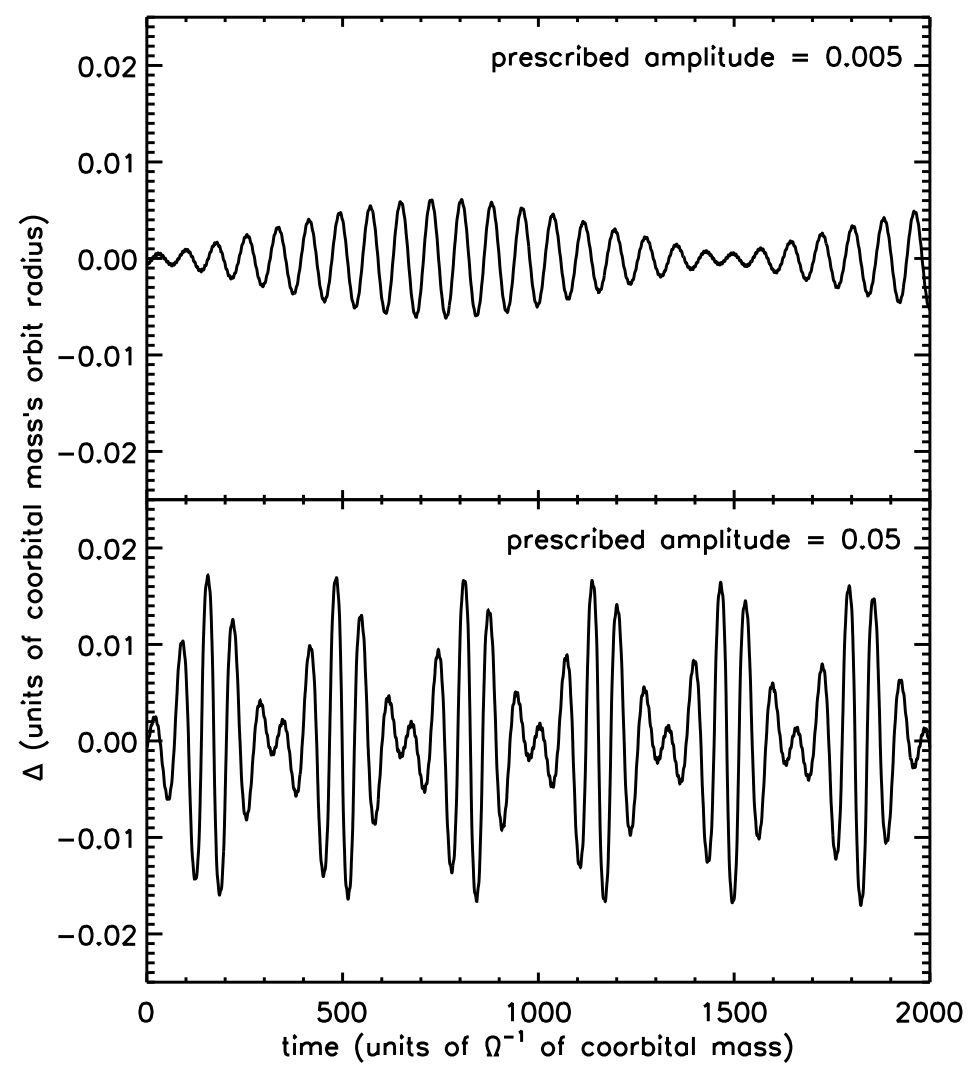

Fig. 4.- Sample moonlet trajectories from experiments where the co-orbital masses move sinusoidally with prescribed amplitude and period. For both panels, the prescribed period is 81.6 (equal to the period that the moonlet would have if the co-orbital masses were stationary). The top panel shows the moonlet's motion when the co-orbital masses move with amplitude 0.005 and the bottom panel shows results when that amplitude is 0.05 . The stronger driving in the bottom panel trajectory leads to both a larger beat amplitude and a higher beat frequency. The latter implies that the co-orbital masses' motion changes the potential seen by the moonlet and the natural frequency of oscillations in that potential. 
damping timescale, dropping factors of order unity. According to the standard impulse approximation (see, for example, Dermott (1984) for a derivation using the impulse approximation or Goldreich \& Tremaine (1982) for a derivation using Lindblad resonances), the moonlet's azimuthal acceleration due to its "one-sided" interaction with ring material on one side of its gap is of order

$$
F_{\theta, 1-\text { sided }} \sim \frac{\mu_{\text {moon }} \Sigma}{x^{3}} .
$$

This is consistent with the scaling $\Delta J \propto \mu_{\text {moon }}^{2} / x^{5}$ for the one-sided impulse of angular momentum per unit disk mass received by the moonlet (see, e.g., Eq. 32 of Crida et al. (2010): multiply their equation by the disk mass $\sim \Sigma x^{2}$ that passes conjunction with the moonlet per unit time - this is analogous to the integral over $x$ that they describe with their Eq. 36 - and divide by $\mu_{\text {moon }}$ to get an acceleration rather than a force). Note that we use $x$ here rather than $x_{\text {gap }}$ because we are concerned with ring material that just passes conjunction with the moonlet, i.e., ring material near the moonlet's longitude.

The moonlet interacts simultaneously with ring material radially interior and exterior to it. If we assume the ring surface density is uniform, then the two one-sided torques cancel up to any asymmetry in the moonlet's radial distances to the gap edges. Then the moonlet's net acceleration is

$$
F_{\theta} \sim-\frac{\Delta}{x} \cdot F_{\theta, 1-\text { sided }} \sim-\frac{\mu_{\text {moon }} \Sigma \Delta}{x^{4}} .
$$

The minus sign enters because the moonlet is repelled more by whichever gap edge is closer to it. The damping timescale is

$$
t_{\text {damp }} \sim\left|\frac{\dot{\theta}}{F_{\theta}}\right| \sim \frac{x^{4}}{\mu_{\text {moon }} \Sigma}
$$

where we have used $|\Delta| \sim|\dot{\theta}|$ due to Keplerian shear (see Eq. (1).

We combine Lindblad damping with co-orbital feedback by adding the term $F_{\theta}$ (as given by Eq. 10, with an assumed coefficient of unity) to the moonlet's azimuthal equation of motion in the numerical toy model of Section 2.1. We expect that damping will balance the (positive) feedback when $t_{\text {damp }}$ is of order the libration growth timescale. In the example shown in the top panel of Figure 3, the timescale to amplify librations is about $t_{\text {amplify }}=$ 6 libration periods of the undriven system (driving alters the potential and shortens the libration period). To check quickly that we understand our simulations in the bottom panel of that figure, we make a simple order-of-magnitude estimate. We set $t_{\text {damp }}=t_{\text {amplify }}=6 P_{\text {lib }}$ and substitute Eqs. 4, 11, and the parameters of the example in Figure 3: $\mu_{\text {end }}=10^{-4.5}$, $x=6\left(\mu_{\text {moon }} / 3\right)^{1 / 3}$ or six Hill radii of the moonlet, $\Sigma=0.01$, and $\phi=0.4$. In this example, damping balances growth when $\mu_{\text {moon }} \sim\left(3^{5 / 2} \pi^{3} / 6^{9}\right) \cdot \phi^{9 / 2} \Sigma^{3} \mu_{\text {end }}^{-3 / 2} \sim 4 \times 10^{-6}$. Our simulations 
show that a somewhat smaller $\mu_{\text {moon }} \sim 7.5 \times 10^{-7}$ is needed (bottom panel of Figure 3), but the agreement is reasonable given the order-of-magnitude nature of our arguments.

Since our Eqs. 10 through 11 neglect all order-unity coefficients — strictly speaking, they are scaling relations - we may apply them to real systems only with the understanding that numerical results cannot be more than broadly suggestive. If we use $x \sim 6$ moonlet Hill radii as before, then Eqs. 4, 11, and the equality in Eq. $7\left(x_{\text {gap }} / \Sigma \sim 24 / \pi^{2}\right)$ give

$$
\frac{t_{\mathrm{damp}}}{P_{\mathrm{lib}}} \sim 0.6\left(\frac{\mu_{\mathrm{moon}}}{3 \times 10^{-15}}\right)^{1 / 3}\left(\frac{\phi}{0.004}\right)^{-1}
$$

where the numerical values are inspired by Blériot (Tiscareno et al. 2010). This numerical result suggests that positive co-orbital feedback and damping Lindblad torques might plausibly balance for propellers in the outer A ring. Unfortunately, the result relies on Eq. 7 , which as we have seen previously demands an unrealistically narrow gap.

\section{Summary and Discussion}

As a step toward a self-consistent model for propellers' non-Keplerian motions, we added to the frog libration model of PC new terms representing co-orbital mass motion and Lindblad torques. In numerical experiments with this 'extended' frog model, we found that allowing the co-orbital mass at the ends of the gap to move in response to the moonlet's non-Keplerian motions can either damp the frog librations completely or drive the librations resonantly. In other words, in our simple model for co-orbital feedback, the feedback is either strongly negative or strongly positive. We found further that the strong positive feedback could be limited, and the motion stabilized, by Lindblad torques.

Our numerical experiments emphasize simplicity over realism. Although they clarify the conditions necessary for stable frog librations, they offer no explanation for why these conditions should be met. That is, we leave unanswered the question of why $t_{\text {delay }}, P_{\text {lib }}$, $\mu_{\text {moon }}$, and $\mu_{\text {ring }}$ of real propeller systems should occur in the right proportions for stable frog librations to exist.

Still, our extended frog model makes observationally testable predictions: if propeller moonlets are performing frog librations, we expect that 1) the properties of Blériot's gap (its azimuthal and radial dimensions, and the co-orbital surface density outside the gap) are such that its frog libration period $P_{\text {lib }} \simeq 4 \mathrm{yr} ; 2$ ) Blériot's and other propellers' longitude residuals will continue to vary sinusoidally in time; 3 ) at any given time, propeller positions will be typically offset from the centers of their gaps by azimuthal distances of order the observed rms longitude residuals; and 4) the properties of propeller gaps are such that gap 
drift (equivalently, gap closing) timescales $t_{\text {delay }}$ (Eq. 5) are comparable to or longer than frog libration periods $P_{\text {lib }}$. Of these four predictions, the first three are retained from PC's original frog model, while the fourth is new from the extended frog model.

Unfortunately, we cannot seem to satisfy both predictions 1 and 4 for Blériot. If we take the azimuthal length of Blériot's gap to be $\phi \approx 0.004$ and its radial width $x_{\text {gap }}$ to be a few $\mathrm{km}$

(a few moonlet Hill radii), we can indeed reproduce $P_{\text {lib }} \simeq 4 \mathrm{yr}$, in accord with the Cassini observations (PC, see the discussion following their Eq. 12). But a gap of such dimensions would close in a time $t_{\text {delay }} \sim 0.01 P_{\text {lib }}$ (Eq. 5) and would lead to a libration amplitude much smaller than the $260-\mathrm{km}$ amplitude that is observed. For the gap drift time $t_{\text {delay }}$ to be longer than the frog libration time $P_{\text {lib }}$, the radial width of the gap may need to be unrealistically small - comparable to the ring particle size. This conclusion is tentative because our treatment of feedback is primitive and possibly oversimplified. We state this shortcoming of our model in the hope that more accurate studies - e.g., numerical simulations designed to explore long-range interactions between the moonlet and the entire gap - may either confirm that the frog resonance is not responsible for the observed non-Keplerian motions, or reveal that feedback works in a way different from what we have imagined in this paper.

We thank an anonymous referee for a detailed reading of this work.

\section{REFERENCES}

Colwell, J. E., Nicholson, P. D., Tiscareno, M. S., Murray, C. D., G., F. R., \& Marouf, E. A. 2009, in Saturn from Cassini-Huygens, ed. M. Dougherty, L. Esposito, \& T. Krimigis (Heidelberg: Springer), 375-412

Crida, A., Papaloizou, J. C. B., Rein, H., Charnoz, S., \& Salmon, J. 2010, AJ, 140, 944

Cuzzi, J., Clark, R., Filacchione, G., French, R., Johnson, R., Marouf, E., \& Spilker, L. 2009, Ring Particle Composition and Size Distribution, ed. Dougherty, M. K., Esposito, L. W., \& Krimigis, S. M., 459-+

Dermott, S. F. 1984, Dynamics of narrow rings, ed. R. Greenberg \& A. Brahic, 589-637

Goldreich, P., \& Tremaine, S. 1982, ARA\&A, 20, 249

Lewis, M. C., \& Stewart, G. R. 2009, Icarus, 199, 387

Pan, M., \& Chiang, E. 2010, ApJ, 722, L178 
Seiß, M., Spahn, F., Sremčević, M., \& Salo, H. 2005, Geophys. Res. Lett., 32, 11205

Spahn, F., \& Sremčević, M. 2000, A\&A, 358, 368

Sremčević, M., Schmidt, J., Salo, H., Seiß, M., Spahn, F., \& Albers, N. 2007, Nature, 449, 1019

Sremčević, M., Spahn, F., \& Duschl, W. J. 2002, MNRAS, 337, 1139

Tiscareno, M. S., Burns, J. A., Hedman, M. M., \& Porco, C. C. 2008, AJ, 135, 1083

Tiscareno, M. S., Burns, J. A., Hedman, M. M., Porco, C. C., Weiss, J. W., Dones, L., Richardson, D. C., \& Murray, C. D. 2006, Nature, 440, 648

Tiscareno, M. S., Burns, J. A., Sremčević, M., Beurle, K., Hedman, M. M., Cooper, N. J., Milano, A. J., Evans, M. W., Porco, C. C., Spitale, J. N., \& Weiss, J. W. 2010, ApJ, 718, L92 